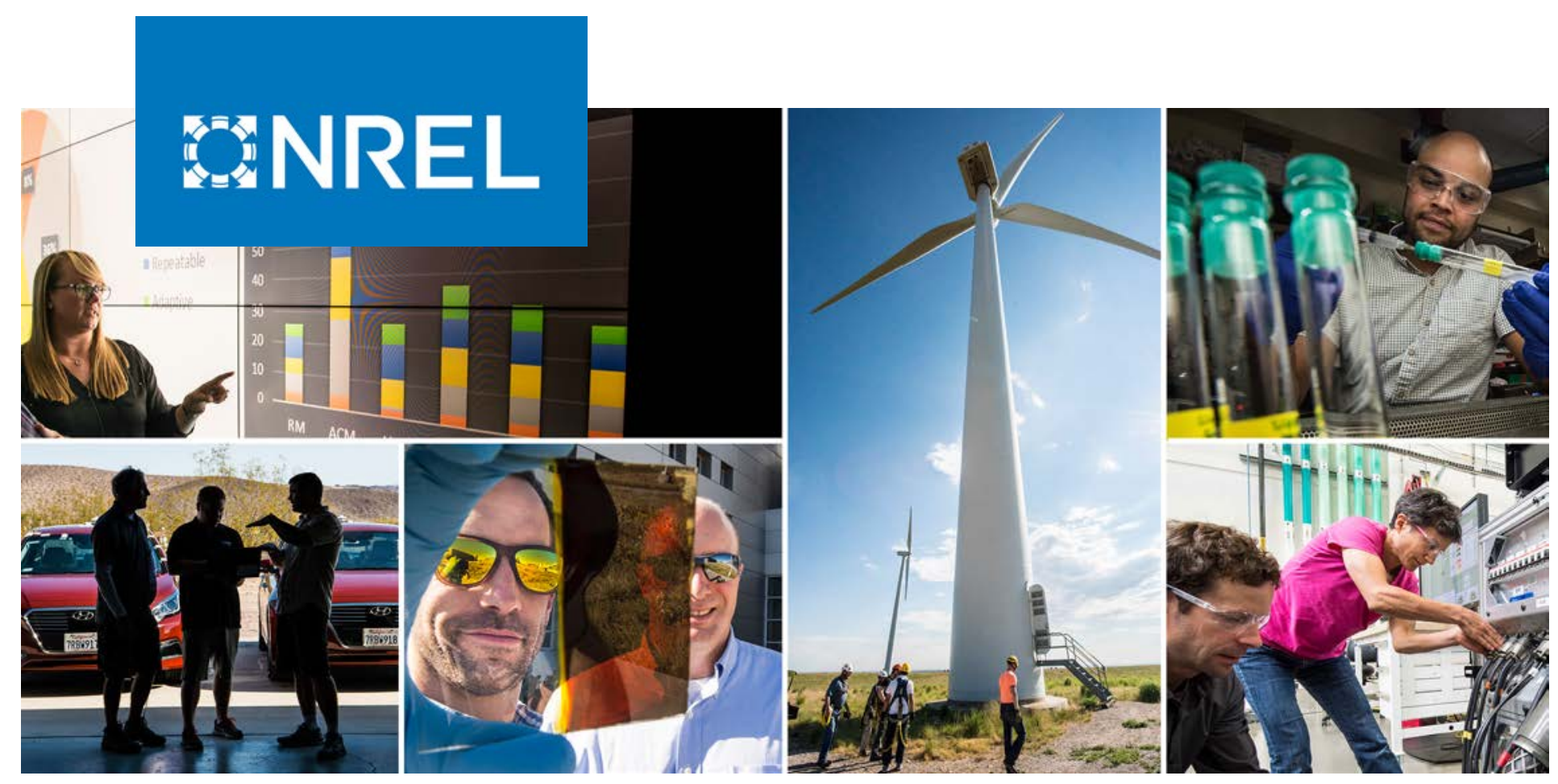

Real-World Distribution System Modeling Framework for Transmission-andDistribution Cosimulation

\title{
Preprint
}

Xiangqi Zhu, ${ }^{1}$ Michael Emmanuel, ${ }^{1}$ Julieta Giraldez, ${ }^{1}$ Ibrahim Krad, ${ }^{1}$ Wenbo Wang, ${ }^{1}$ Bryan Palmintier, ${ }^{1}$ Wei-Hann Chen, ${ }^{2}$ Alan Hirayama, ${ }^{2}$ and Marc Asano ${ }^{2}$

${ }^{1}$ National Renewable Energy Laboratory

${ }^{2}$ Hawaii Electric

Presented at the 47th IEEE Photovoltaic Specialists Conference (PVSC 47) June 15-August 212020

NREL is a national laboratory of the U.S. Department of Energy

Office of Energy Efficiency \& Renewable Energy

Operated by the Alliance for Sustainable Energy, LLC

This report is available at no cost from the National Renewable Energy Laboratory (NREL) at www.nrel.gov/publications. 


\title{
GNREL
}

\section{Real-World Distribution System Modeling Framework for Transmission-and- Distribution Cosimulation}

\section{Preprint}

\author{
Xiangqi Zhu, ${ }^{1}$ Michael Emmanuel, ${ }^{1}$ Julieta Giraldez, ${ }^{1}$ \\ Ibrahim Krad, ${ }^{1}$ Wenbo Wang, ${ }^{1}$ Bryan Palmintier, ${ }^{1}$ \\ Wei-Hann Chen, ${ }^{2}$ Alan Hirayama, ${ }^{2}$ and Marc Asano ${ }^{2}$ \\ ${ }^{1}$ National Renewable Energy Laboratory \\ ${ }^{2}$ Hawaii Electric
}

\section{Suggested Citation}

Zhu, Xiangqi, Michael Emmanuel, Julieta Giraldez, Ibrahim Krad, Wenbo Wang, Bryan Palmintier, Wei-Hann Chen, Alan Hirayama, and Marc Asano. 2020. Real-World Distribution System Modeling Framework for Transmission-and-Distribution Cosimulation: Preprint. Golden, CO: National Renewable Energy Laboratory. NREL/CP-5D00-74839. https://www.nrel.gov/docs/fy20osti/74839.pdf.

(C) 2020 IEEE. Personal use of this material is permitted. Permission from IEEE must be obtained for all other uses, in any current or future media, including reprinting/republishing this material for advertising or promotional purposes, creating new collective works, for resale or redistribution to servers or lists, or reuse of any copyrighted component of this work in other works.

NREL is a national laboratory of the U.S. Department of Energy Office of Energy Efficiency \& Renewable Energy Operated by the Alliance for Sustainable Energy, LLC

This report is available at no cost from the National Renewable Energy Laboratory (NREL) at www.nrel.gov/publications.

Contract No. DE-AC36-08G028308
Conference Paper NREL/CP-5D00-74839 August 2020

National Renewable Energy Laboratory 15013 Denver West Parkway Golden, CO 80401 303-275-3000 • www.nrel.gov 


\section{NOTICE}

This work was authored by the National Renewable Energy Laboratory, operated by Alliance for Sustainable Energy, LLC, for the U.S. Department of Energy (DOE) under Contract No. DE-AC36-08GO28308. Funding provided by U.S. Department of Energy Office of Energy Efficiency and Renewable Energy Solar Energy Technologies Office via the Grid Optimization with Solar project. The views expressed herein do not necessarily represent the views of the DOE or the U.S. Government.

This report is available at no cost from the National Renewable Energy Laboratory (NREL) at www.nrel.gov/publications.

U.S. Department of Energy (DOE) reports produced after 1991 and a growing number of pre-1991 documents are available free via www.OSTI.gov.

Cover Photos by Dennis Schroeder: (clockwise, left to right) NREL 51934, NREL 45897, NREL 42160, NREL 45891, NREL 48097, NREL 46526.

NREL prints on paper that contains recycled content. 


\section{Real-World Distribution System Modeling Framework for Transmission-and-Distribution Cosimulation}

\author{
Xiangqi Zhu, Michael Emmanuel, Julieta Giraldez, \\ Ibrahim Krad, Wenbo Wang, and Bryan Palmintier \\ Power Systems Engineering Center \\ National Renewable Energy Laboratory \\ Golden, CO, USA \\ xiangqi.zhu@nrel.gov
}

\author{
Wei-Hann Chen, Alan Hirayama, Marc Asano \\ Hawaii Electric \\ Honolulu, Hawaii, USA \\ weihann.chen@hawaiianelectric.com
}

\begin{abstract}
This paper presents a modeling framework for realworld distribution systems to enable large-scale transmission-anddistribution (T\&D) cosimulation. The modeling methodology includes three major steps: utility feeder model conversion, feeder load modeling, and model validation. The feeder models obtained from the utility are converted to the format that is more flexible for analysis and algorithm development. The load profile of each medium-voltage bus is modeled based on the data obtained from advanced metering infrastructure and the supervisory control and data acquisition (SCADA) system. Then the converted feeder models are validated by comparing the simulated feeder-head voltage results to the measured voltage data from SCADA. Last, the modeled real-world large-scale distribution systems are integrated into a $T \& D$ cosimulation framework to perform the system simulation from the bulk system down to the mediumvoltage buses in the distribution system.
\end{abstract}

Keywords-distribution system; model conversion; load modeling; model validation; $T \& D$ cosimulation

\section{INTRODUCTION}

With the anticipated increasing penetration levels of renewable generation on the electric power grid, utilities are actively seeking advanced system analysis and control methodologies to tackle the challenges brought on by renewable generation integration. As an important cornerstone, realistic and accurate system modeling sets the base for system simulation, scenario design, problem analysis, and control algorithm development and testing.

Moreover, renewable energy resources hosted by distribution systems are becoming promising candidates to provide grid services on both the transmission and distribution (T\&D) levels. Hence, realistic system modeling is needed that could enable T\&D cosimulation and T\&D hierarchical control development and testing. Because distribution systems serve as direct connections to distributed energy resources (DERs), successful and realistic modeling of distribution systems is critical for integrated T\&D cosimulation and control development.
Realistic distribution system modeling generally involves three parts: system parameter and configuration modeling, load modeling, and DER modeling. Currently, distribution system modeling methods in the literature mostly focus on different load and DER modeling approaches for system planning, reliability analysis, demand response, etc. [1]-[5]; however, system parameter and configuration modeling are barely considered. Moreover, few studies provide a systematic framework for distribution system modeling to string together all the parts, including system parameter and configuration modeling as well as load and DER modeling. Modeling a realistic distribution system, especially modeling distribution systems using data provided by utilities, requires effective modeling of system parameters and configuration for control scenario design and system analysis. A systematic distribution system modeling method that could consider all the parts could greatly help modelers and researchers use the modeling efforts wisely and effectively.

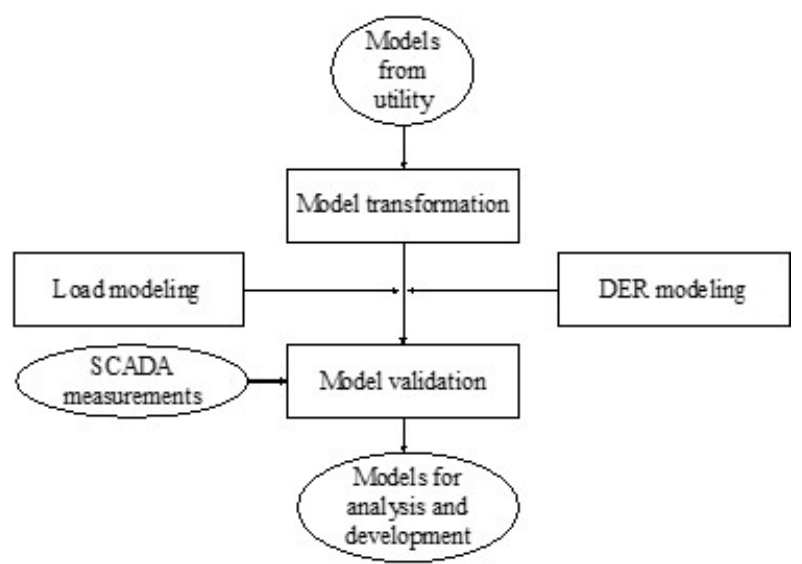

Fig. 1. Systematic method for distribution system modeling

In this work, we develop a systematic framework for distribution system modeling. As shown in Fig. 1, the system models obtained from the utility are first converted to the format that is friendly to research analysis and control 
algorithm development, then the load and DERs are modeled and added to the system models. Finally, the developed models with loads and DERs are validated against the supervisory control and data acquisition (SCADA) system measurements to ensure that future analysis and control algorithms developed on these models represent realistic conditions. This paper focuses on discussing the model transformation, load modeling, and model validation. DER modeling will be discussed in our follow-up paper.

The developed systematic methodology is applied to modeling the distribution systems on one of the Hawaiian Islands. To better integrate the distribution system models into the T\&D framework, the same methodology is applied to model the subtransmission systems. The model validation and testing results demonstrate that the developed realistic distribution system models well capture the system parameters and load characteristics.

The rest of this paper is organized as follows: Section II introduces the modeling methodology, including utility model conversion, load modeling, and feeder model validation. This section also introduces the T\&D cosimulation framework into which the modeled distribution and subtransmission systems are integrated. Section III presents the case study performed on the Hawaiian distribution systems. Section IV concludes and discusses future work.

\section{Modeling Methodology}

This section introduces the systematic framework we developed for real-world distribution system modeling. Four steps are introduced successively:

1) Utility model conversion, in which we convert the distribution system models from operation-oriented formats to research-oriented formats

2) Feeder load modeling, in which we model the load profiles along the feeder

3) Feeder model validation, in which we validate the modeled system against the SCADA measurements

4) $T \& D$ cosimulation, in which we integrate the distribution system models into a T\&D cosimulation framework.

\section{A. Utility Model Conversion}

Usually, distribution system models directly obtained from utilities are in the formats that are suitable only for specific software used by utilities (e.g., Synergi, Cyme, Windmil); however, these software are not flexible for research and algorithm development. Therefore, utility formats are first converted to an open-source simulator software format, such as OpenDSS or GridLAB-D.

Because the data set is large, it is necessary to have an automated process for the conversion. The Distribution Transformation Tool (DiTTo) [6] is leveraged to perform the conversion. DiTTo is a Python-based open-source model conversion tool that can convert different formats of distribution system models into a number of formats. Further, the resulting data formats need to be stored in a way that is flexible and convenient for cosimulation; each distribution substation should have its own directory so that they can be run not only together but also separately. Further, within each substation directory, there are feeders that belong to the substation that can also be run together and separately. Through this hierarchical arrangement, different scales of system simulations (i.e., network resolution) can be enabled.

An example of this conversion process is shown in Fig. 2. The model obtained from the utility is in Synergi format, including subtransmission and feeder models. In this case, the utility models are converted to OpenDSS using DiTTo. The resulting data are stored in such directories so that the models are immediately ready for different scales of simulations.

This automated flow enables time-effective modeling for large-scale distribution systems (i.e., 1 million medium-voltage buses). In this paper, we take a smaller system as an example for illustration.

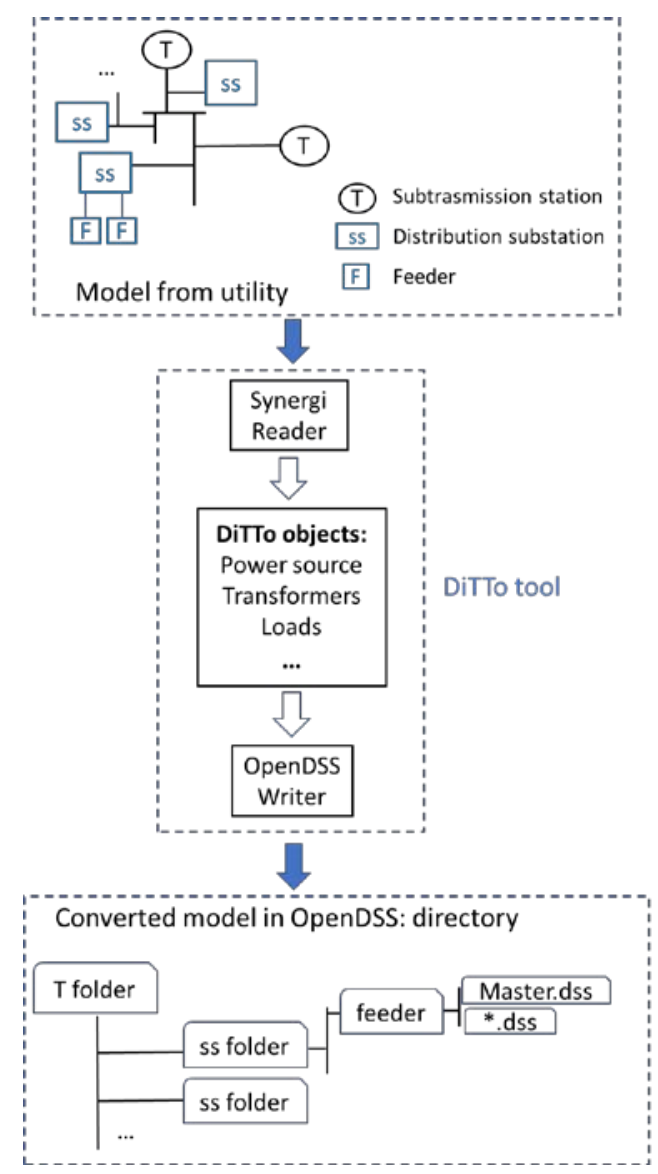

Fig. 2. DiTTo conversion example

\section{B. Feeder Load Modeling}

Most utilities do not have or are in the process of deploying advanced metering infrastructure (AMI) data, and distribution modeling and simulation studies leverage SCADA substation data to approximate the same load profile for all customers. It is increasingly important, however, to capture and represent the load diversity to create more detailed distribution system models. This study leverages smart meter data for more than 3,500 customers in Hawaii and applies clustering techniques to 
identify representative load profiles from which to build more diverse aggregate load profiles to feed into distribution system models. As shown in Fig. 3, a symbolic aggregate approximation-based dimensionality-reduction and k-means techniques (Fig. 3 (a)) are used to identify typical load profiles (Fig. 3(b)) [7]. The representative profiles are then selected and used to estimate the aggregated load profiles at the distribution service transformer level based on the number of customers connected to each transformer.

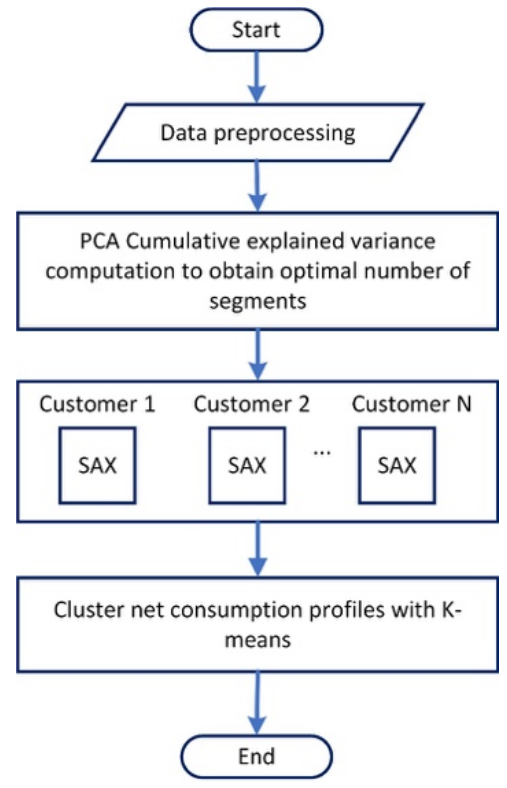

(a) Load modeling flowchart

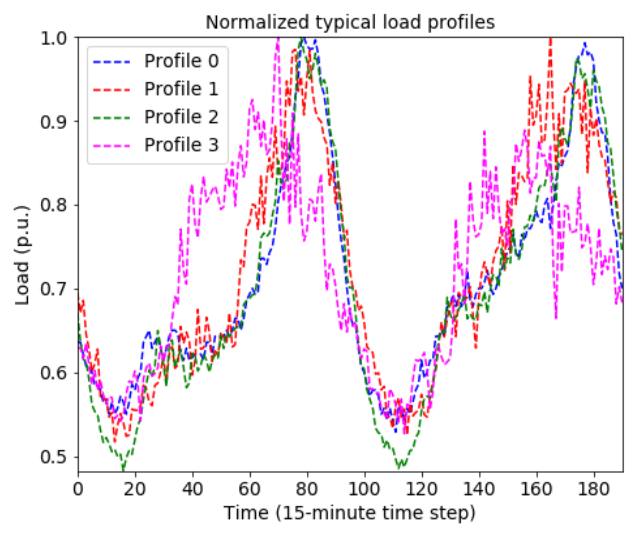

(b) Sample typical load profiles

Fig. 3. Load modeling method overview

After the load profiles are generated, a load aggregation and scaling method developed in our previous work [8]-[9] is used to ensure that the summation of the load profiles in a feeder matches the SCADA measured load profile.

\section{Model Validation}

As shown in Fig. 4, the SCADA measured feeder-head load profiles are disaggregated to each node using the method mentioned in Part B of Section II. In this way, load nodes along the feeder have a diversified load shape, and the summation of the load profiles matches the SCADA load profile. Then a set of voltage results is obtained after running a time-series power flow case.

The developed distribution models are validated by comparing the simulated voltage profile of each phase to the SCADA measured voltage profile that is associated with the measured feeder-head load profiles. The error rate $r(t)$ is calculated using (1)-(2). Based on communications with utility partners, we are confident that the model could maintain high fidelity if the average error rate, $r_{\text {mean }}$, is less than $1 \%$.

$$
\begin{gathered}
r(t)=\frac{\left|V_{\text {simu }}(t)-V_{\text {SCADA }}(t)\right|}{V_{S C A D A}(t)} \\
r_{\text {mean }}=\frac{\sum_{t=1}^{n} r(t)}{n}
\end{gathered}
$$

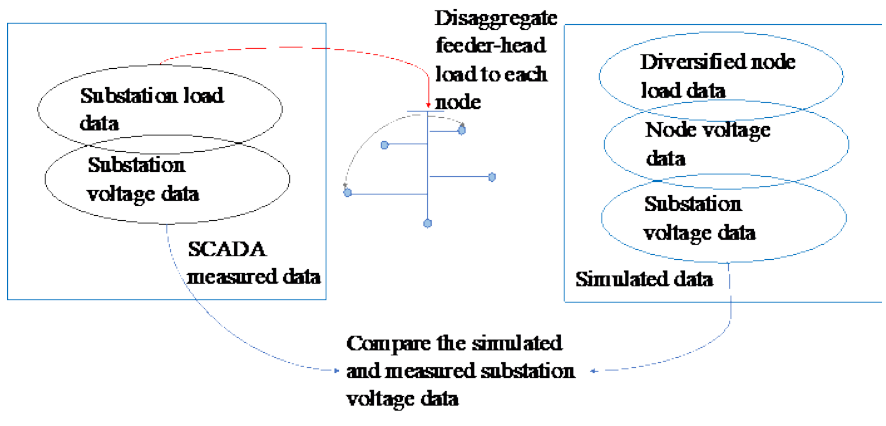

Fig. 4. Model validation process

\section{T\&D Cosimulation}

The cosimulation leverages the Integrated Grid Modeling System (IGMS) and Hierarchical Engine for Large-Scale Infrastructure Co-Simulation (HELICS) [10]-[11]. As shown in Fig. 5, this cosimulation workflow provides a loosely coupled solution algorithm to iterate between each individual model until the simulation time is complete. The transmission simulation provides generator set points (economic dispatch) and then solves an AC power flow. The solution of the power flow (voltage) is passed to the distribution models as the voltage on the high side of distribution feeder heads. The distribution feeder models accept this voltage and perform distribution power flow. The output of this distribution power flow (load/appliance set points) is then aggregated and sent back to the transmission model. This entire iteration occurs during one simulation time step, which is typically every $4-6$ seconds. The communications and passing of data between the different models are choreographed by HELICS [10]-[11], which is the cosimulation engine leveraged by IGMS. 


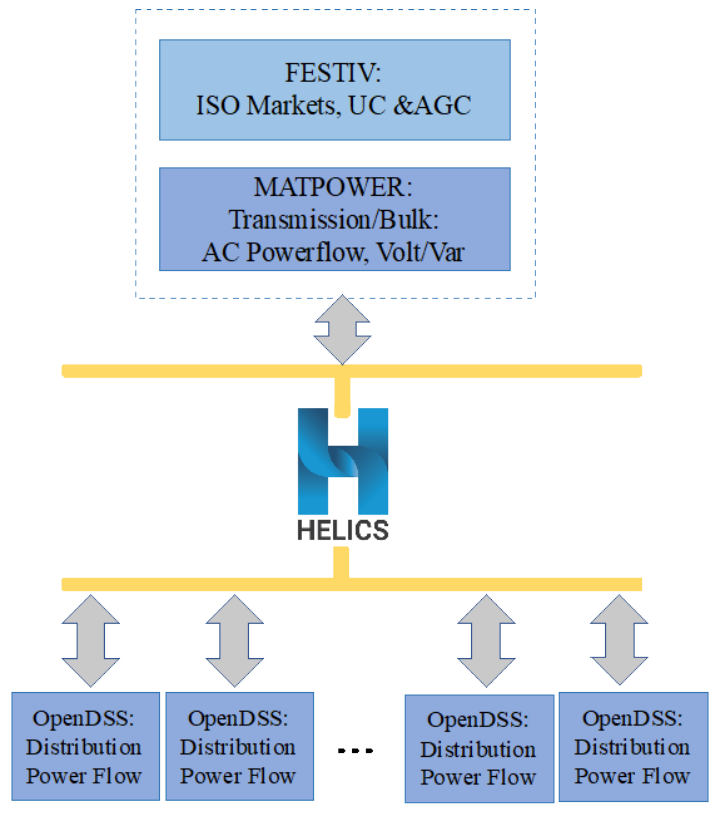

Fig. 5. T\&D cosimulation framework

\section{CASE Study On Hawail FEeders}

This section introduces the modeling case for a Hawaiian island. To explore the pathway of modeling the whole island in realistic detail, we model a reduced T\&D model that includes a full set of transmission system models and a subset of subtransmission and distribution system models. This section focuses on modeling the subtransmission and distribution systems.

\section{A. System Models}

As shown in Fig. 6, a top-down approach is used in this case. The transmission system is modeled first, with each load bus (i.e., subtransmission station, $138 / 46 \mathrm{kV}$ connecting a lumped load. Then one representative bus is selected for modeling the downstream subtransmission and distribution systems in detail; the selected bus is connected to two subtransmission transformers, with each transformer connecting with several distribution systems.

Starting with the two subtransmission station transformers, a set of substation transformers (e.g., $46 / 12.47 \mathrm{kV}$ at the distribution level is connected to the two subtransmission station transformers through underground and overhead lines. Each substation transformer connects with one or two distribution feeders. Four representative feeders are selected for modeling the downstream lines and loads in detail, and the other feeders are modeled as lumped loads connected to the substation transformers.

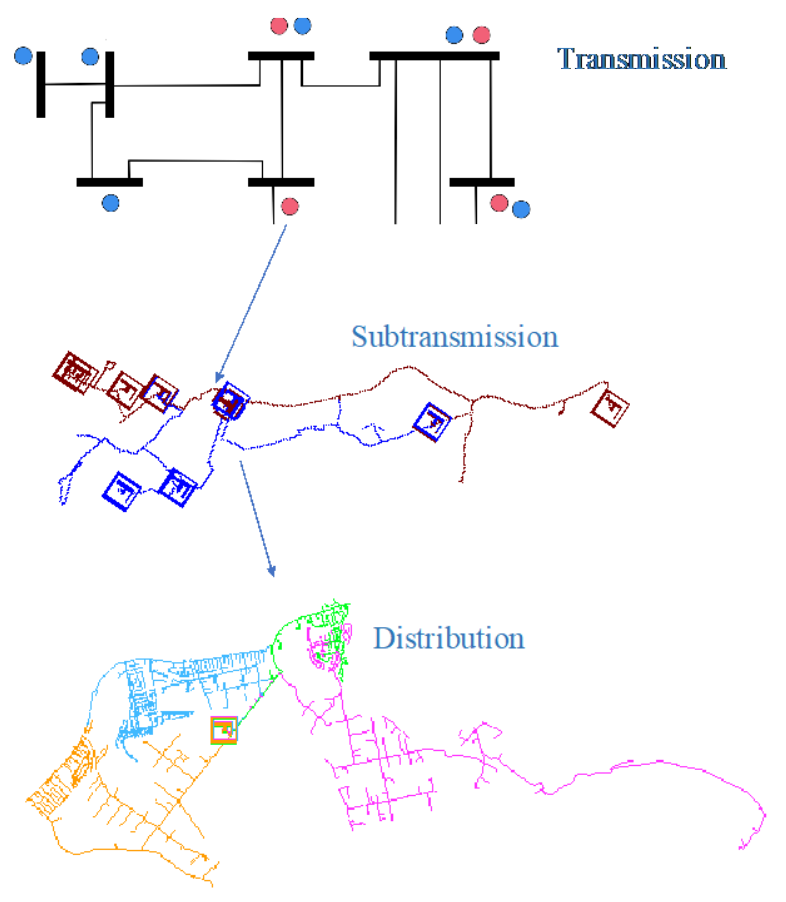

Fig. 6. System modeling structure

\section{B. Load Profile Generation}

The load profiles in the four selected distribution feeders are generated using the method discussed in Section II. Each of the four feeders has a time-series feeder-head profile obtained from the SCADA measurements. AMI data are used to generate the load profiles on the medium-voltage buses.

As shown in Fig. 7 (a), the load profiles with the feeder nodes are diversified and have different shapes, whereas the two profiles shown in Fig. 7(b) demonstrate that the feederhead load profiles match the SCADA measurements.

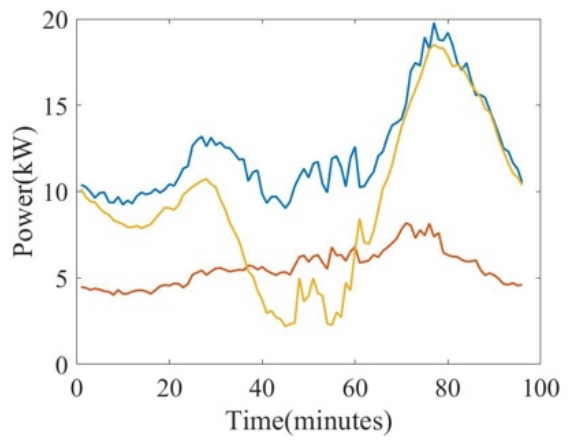

(a) Node load profile samples 


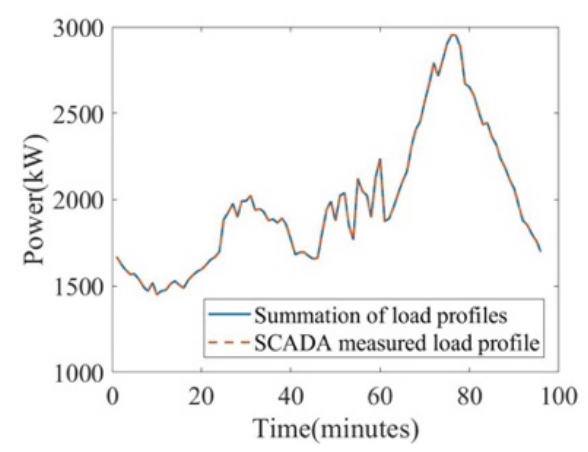

(b) Feeder-head load profile comparison

Fig. 7. Load profiles

\section{Model Validation}

The distribution system models are validated using the method presented in Section II. The subtransmission and transmission model validations will be introduced in our follow-up paper.

Fig. 8 illustrates the validation process. Because of the lack of voltage measurements at the feeder head, which is the secondary side of the substation transformer, the measured voltage data at the subtransmission transformer secondary side are used as input data to perform the subtransmission system simulation. Then the voltage data at the secondary side of the corresponding substation transformers are used as measurements for the validation. Here, lumped loads are connected to the distribution transformers, with load profiles obtained from SCADA load measurements.

The voltage at primary side of the substation transformers is used as input data to perform the distribution system simulation; the detailed feeder model starts with the substation transformers and goes down to the medium-voltage buses instead of modeling an entire feeder as lumped loads.

To validate that the system parameters and load models of the distribution systems can well represent the SCADA measured load characteristics at the feeder head, the voltage data at the secondary side of the substation transformers in the distribution system simulation are validated against those in the subtransmission system simulation, as shown in Fig. 8.

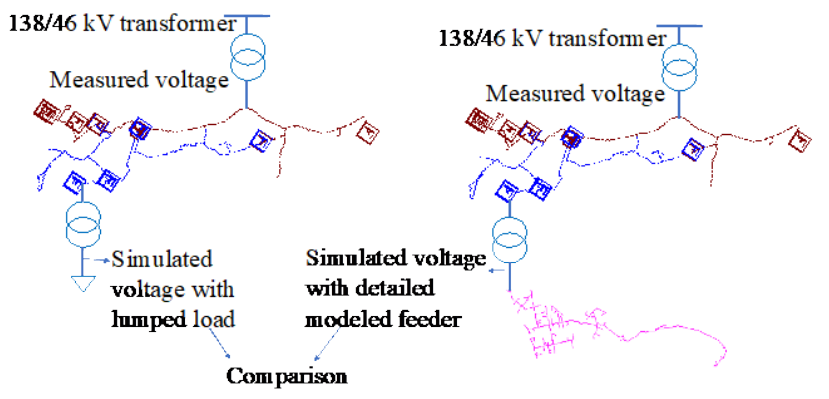

Fig. 8. Model validation structure

The validation results for one of the distribution systems are shown in Fig. 9 and Fig. 10. The voltage profile comparisons of the three phases are shown in Fig. 9 (a)-(c). The average voltage profile of the three phases are shown in Fig. 9 (d). Fig. 10 shows the histogram of the error rate, which is calculated using (1) in Section II. Fig. 10 shows that the maximum error rate is less than $0.5 \%$, which well demonstrates that the detailed feeder models can capture the load and feeder characteristics of the SCADA measurements.

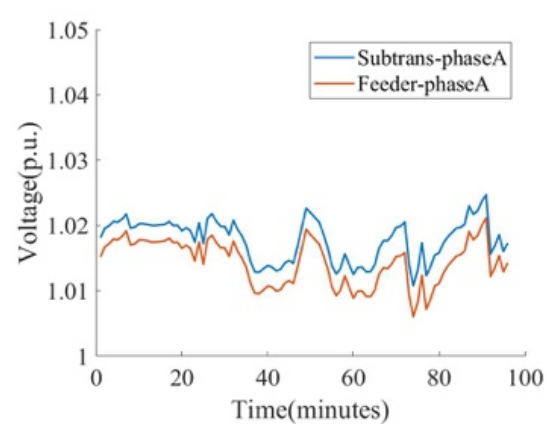

(a)

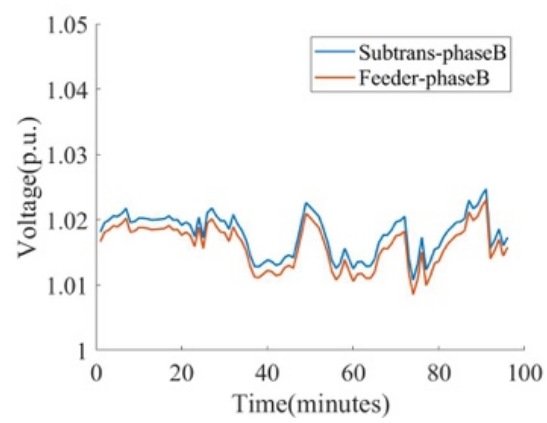

(b)

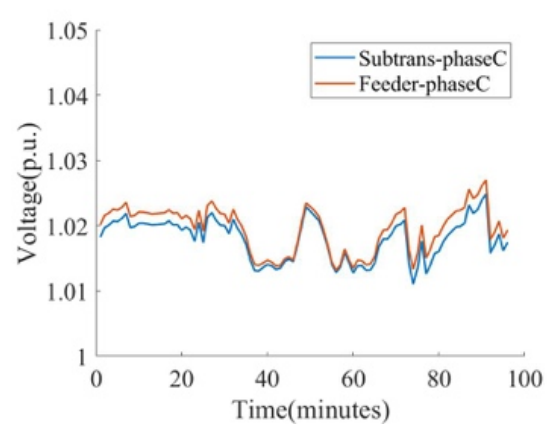

(c)

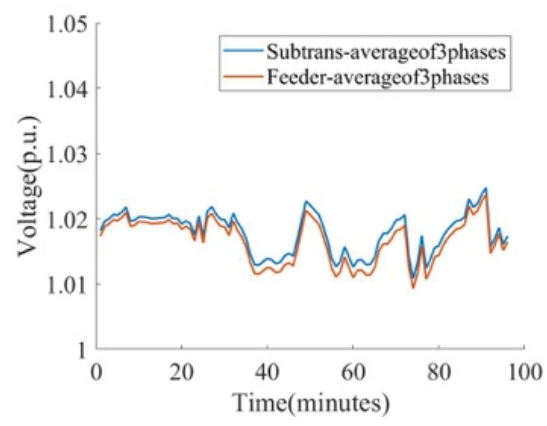

(d)

Fig. 9. Voltage profile comparison 


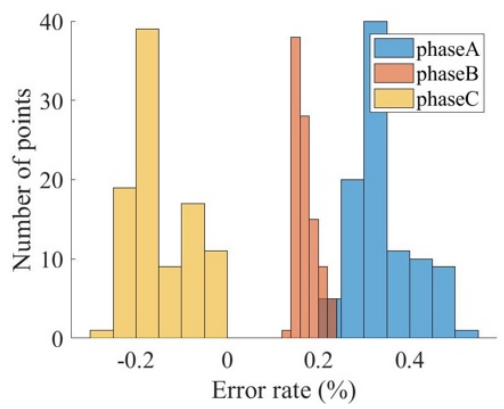

Fig. 10. Error rate distribution

\section{CONCLUSIONS AND FUTURE WORK}

This paper presented a systematic framework for modeling real-world distribution and subtransmission systems aiming at integrated $\mathrm{T} \& \mathrm{D}$ cosimulation and system analysis. This approach uses real data supplied by the utility and comprises three major steps: utility feeder model conversion, load profile generation, and model validation. Using these three steps, realistic distribution and subtransmission systems can be modeled in a format that is flexible for analysis and algorithm development and testing. Diversified load profiles generated from measured AMI data are equipped at each load bus, with the summation of all the load profiles along a feeder matching the SCADA-measured feeder-head load profile. The validated models can be integrated into the T\&D cosimulation framework for system analysis and algorithm testing.

Following the work presented, we plan to develop a delicate algorithm to enable situation awareness of distribution system connection with subtransmission systems, as well as the AMI meter connection with feeder-load buses, to improve the modeling accuracy. In addition, we will consider the system losses when modeling the load profiles of the feeders. Further, we will work with the utility partner to accurately and realistically model the system photovoltaic generation.

\section{ACKNOWLEDGMENTS}

This work was authored by Alliance for Sustainable Energy, LLC, the manager and operator of the National Renewable Energy Laboratory for the U.S. Department of Energy (DOE) under Contract No. DE-AC36-08GO28308. Funding provided by U.S. Department of Energy Office of Energy Efficiency and Renewable Energy, Solar Energy Technologies Office via the Grid Optimization with Solar project. The views expressed in the article do not necessarily represent the views of the DOE or the U.S. Government. The U.S. Government retains and the publisher, by accepting the article for publication, acknowledges that the U.S. Government retains a nonexclusive, paid-up, irrevocable, worldwide license to publish or reproduce the published form of this work, or allow others to do so, for U.S. Government purposes.

\section{REFERENCES}

[1] Kersting, William H. Distribution system modeling and analysis. CRC press, 2006.

[2] Qian, Kejun, Chengke Zhou, Malcolm Allan, and Yue Yuan. "Modeling of load demand due to EV battery charging in distribution systems." IEEE Transactions on Power Systems26, no. 2 (2010): 802-810.

[3] Ghosh, Atish K., David L. Lubkeman, and Robert H. Jones. "Load modeling for distribution circuit state estimation." IEEE Transactions on Power Delivery 12, no. 2 (1997): 999-1005.

[4] Wang, Haibin, and Noel N. Schulz. "A load modeling algorithm for distribution system state estimation." In 2001 IEEE/PES Transmission and Distribution Conference and Exposition. Developing New Perspectives (Cat. No. 01CH37294), vol. 1, pp. 102-105. IEEE, 2001.

[5] Wang, Jiyu, Xiangqi Zhu, D. Lubkeman, Ning Lu, Nader Samaan, and Brant Werts. "Load aggregation methods for quasi-static power flow analysis on high PV penetration feeders." In 2018 IEEE/PES Transmission and Distribution Conference and Exposition (T\&D), pp. 1-5. IEEE, 2018.

[6] https://github.com/NREL/ditto

[7] M. Emmanuel and J. Giraldez, "Net Electricity Clustering at Different Temporal Resolutions Using a SAX-Based Method for Integrated Distribution System Planning", IEEE Access, 2019 (Article accepted for publication)

[8] Zhu, Xiangqi, Jiyu Wang, Ning Lu, Nader Samaan, Renke Huang, and Xinda Ke. "A hierarchical vlsm-based demand response strategy for coordinative voltage control between transmission and distribution systems." IEEE Transactions on Smart Grid (2018).

[9] Zhu, Xiangqi, Jiyu Wang, David Mulcahy, David L. Lubkeman, Ning Lu, Nader Samaan, and Renke Huang. "Voltage-load sensitivity matrix based demand response for voltage control in high solar penetration distribution feeders." In 2017 IEEE Power \& Energy Society General Meeting, pp. 1-5. IEEE, 2017.

[10] Palmintier, B., E. Hale, T. M. Hansen, W. Jones, D. Biagioni, H. Sorensen, H. Wu, and B. M. Hodge. 2016. "IGMS: An Integrated ISO-to-Appliance Scale Grid Modeling System." IEEE Transactions on Smart Grid 8 (3): 1525-34. https://doi.org/10.1109/TSG.2016.2604239.

[11] Palmintier, Bryan, Dheepak Krishnamurthy, Philip Top, Steve Smith, Jeff Daily, and Jason Fuller. 2017. "Design of the HELICS High-Performance TransmissionDistribution-Communication-Market Co-Simulation Framework." In Proc. of the 2017 Workshop on Modeling and Simulation of Cyber-Physical Energy Systems. Pittsburgh, PA. 